EDITOR'S

${ }^{1}$ Department of Medicine for the Elderly, Lincoln County Hospital, Lincoln, UK

${ }^{2}$ Division of Geriatric Medicine, Nottingham University, Nottingham, UK

\section{Correspondence to}

Dr Darren Aw, Lincoln County Hospital, Greetwell Road, Lincoln LN2 50Y, UK darren.aw@gmail.com

Received 15 April 2011 Accepted 6 November 2011 Published Online First 25 November 2011

\title{
Antiplatelets in secondary stroke prevention: should clopidogrel be the first choice?
}

\author{
Darren Aw, Jagdish C Sharma ${ }^{1,2}$
}

\section{ABSTRACT}

Antiplatelet therapy has proven efficacy in the secondary prevention of recurrent non-cardioembolic ischaemic stroke. Recent evidence suggests clopidogrel is as effective as combined therapy with aspirin and extendedrelease dipyridamole for the prevention of recurrent stroke. As cerebrovascular and ischaemic heart disease are closely related, it would be sensible to use a drug shown to prevent vascular events in both territories. Clopidogrel meets these criteria, is superior to aspirin monotherapy, and has fewer side effects compared with extended-release dipyridamole. While there is no direct evidence supporting the use of clopidogrel in transient ischaemic attacks, it is likely that clopidogrel is effective because transient ischaemic attacks and stroke are part of the same disease spectrum. Clopidogrel could thus be useful as first line secondary prevention therapy in all non-cardioembolic stroke subtypes and transient ischaemic attacks, to prevent recurrent ischaemic events in all vascular territories.

\section{INTRODUCTION}

Stroke is a major cause of morbidity and mortality. Recurrent ischaemic stroke is preventable, and various evidence-based treatment regimens are available. Treatments should be chosen for both their efficacy and their tolerability. In patients with ischaemic stroke, the efficacy of clopidogrel was recently compared with that of aspirin and extended-release dipyridamole (ER-DP) used in combination, with similar efficacy found for both treatment regimens. ${ }^{1}$ A new drug, cilostazol, has also been shown to be at least as effective as aspirin alone. $^{2}$ As cerebrovascular and ischaemic heart disease frequently occur concurrently in patients with previous ischaemic stroke, it would be sensible to use a drug with established efficacy for treating vascular disease in different territories. In this review, we examine the evidence base for the various antiplatelet therapies currently used in secondary stroke prevention, compare their side effect profiles and discuss the use of current antiplatelets in general atheromatous disease, and in different non-cardioembolic ischaemic stroke subtypes. We also discuss current recommendations set out in the recent guidelines produced by the National Institute for Health and Clinical Excellence (NICE), the European Stroke Organisation and the American Stroke Association.

\section{THE EVIDENCE BASE FOR ANTIPLATELET THERAPY IN SECONDARY STROKE PREVENTION}

Aspirin has long been used for the prevention of recurrent ischaemic stroke, and has been shown in both the Chinese Acute Stroke Trial (CAST) and the International Stroke Trial (IST) to produce a modest benefit, with an $R R$ reduction (RRR) of $13 \%{ }^{3}$ The Antithrombotic Trialists' Collaboration meta-analysis confirmed its role in secondary stroke prevention with an RRR of $22 \%$ in ischaemic stroke events (OR $0.78,95 \%$ CI 0.61 to $0.99 ; p=0.04) .{ }^{4}$ Trials of aspirin versus aspirin plus dipyridamole (European Stroke Prevention Study 2 (ESPS2) $)^{5}$ and the European/Australasian Stroke Prevention in Reversible Ischaemia Trial (ESPRIT) ${ }^{6}$ ) have shown that when ER-DP is combined with aspirin, there is a further significant reduction in recurrent stroke when compared to aspirin or ER-DP alone. Aspirin has also been compared with the thienopyridines ticlopidine and clopidogrel. Two trials have showed that ticlopidine was not superior to aspirin therapy in secondary stroke prevention. ${ }^{7}$ The Clopidogrel versus Aspirin in Patients at Risk of Ischemic Events (CAPRIE) trial showed that clopidogrel was modestly superior to aspirin, with an RRR of $8.7 \%$ in favour of clopidogrel for recurrent events, and in the stroke subgroup there was a trend towards benefit on clopidogrel with an RRR of $7.3 \%$ (95\% CI $-5.7 \%$ to $18.7 \%$; $p=0.26) .{ }^{9}$ A systematic review by the Cochrane Collaboration showed that the thienopyridines were more effective than aspirin for reducing vascular events (OR 0.91, 95\% CI 0.84 to 0.98), as well as ischaemic and all stroke events. ${ }^{10}$ Clopidogrel has also been combined with aspirin in the Management of Atherothrombosis with Clopidogrel in High risk patients (MATCH) ${ }^{11}$ and in the Clopidogrel for High Atherothrombosis Risk and Ischemic Stabilization, Management and Avoidance (CHARISMA) ${ }^{12}$ trials, which showed a non-significant trend towards benefit with the combination therapy, but with an increase in haemorrhagic complications. The Clopidogrel and Aspirin for Reduction of Emboli in Symptomatic Carotid Stenosis (CARESS) trial also examined combination therapy with clopidogrel and aspirin in comparison with aspirin monotherapy in patients with symptomatic carotid stenosis ( $>50 \%$ stenosis), and found that combination therapy was more effective with no differences in bleeding side effects, with an RRR of $39.8 \%$ (95\% CI $13.8 \%$ to $58.0 \% ; p=0.0046)$. However, the primary endpoint was based on a surrogate marker of recurrent stroke risk (microembolic signals measured with transcranial doppler) rather than hard endpoints and the cohort was small. ${ }^{13}$ The ongoing Triple Antiplatelets for Reducing Dependency after Ischaemic Stroke (TARDIS) trial which is also investigating the use of aspirin and clopidogrel but with the addition of dipyridamole, should provide more insight into the use of clopidogrel as part of 
combination therapy for the management of patients at high risk of recurrent stroke. ${ }^{14}$

More recently, the Prevention Regimen for Effectively Avoiding Second Strokes (PRoFESS) trial compared the combination of aspirin and ER-DP versus clopidogrel, and found that both treatment regimens had comparable efficacy, with the combination being similar to clopidogrel (9\% vs 8.8\%, HR 1.01, 95\% CI 0.92 to 1.11$)^{1}$ (table 1 ).

It is worth noting that the majority of patients recruited into the PRoFESS study had lacunar strokes (approximately 52\%), while only $28 \%$ of the strokes were of the large artery subtype. Given that most patients in this study had mild stroke, the overall results could have been skewed. As it is unlikely that a trial with over 20000 patients will be conducted again, and as the present PRoFESS trial is inadequately powered to examine treatment effects in stroke subtypes, it may never be known if clopidogrel is superior to aspirin and ER-DP combination therapy in large artery stroke subtypes. In a previously published hypothetical subgroup analysis, it was found that there was potentially significant heterogeneity in the treatment effects of the two drug regimens on different stroke subtypes (large artery subtype vs lacunar stroke subtype). ${ }^{15}$ The large artery subtype patients had a potentially better benefit with clopidogrel compared with the aspirin and ER-DP combination $(1.1 \%$ absolute risk reduction in recurrent stroke; $p=0.05$ ), while the treatment outcomes were very similar in the lacunar stroke subtype (8.2\% for those on clopidogrel vs $7.9 \%$ for those on combination therapy).

A recent randomised controlled trial has examined cilostazol, a new antiplatelet agent that seems to be at least as effective as aspirin in the prevention of recurrent stroke $(2.76 \%$ for those on cilostazol vs $3.71 \%$ for those on aspirin, HR $0.743,95 \%$ CI 0.564 to 0.981$)$. There were fewer haemorrhagic events in the cilostazol group than the aspirin group $(0.77 \%$ vs $1.78 \%$, HR 0.458 , $95 \%$ CI 0.296 to 0.711$)^{2}$ Future studies comparing cilostazol with various other antiplatelet agents are needed to determine its place in secondary stroke prevention.

\section{THE SIDE EFFECT PROFILE OF VARIOUS ANTIPLATELET DRUGS}

Aspirin, the most commonly used antiplatelet, is well known for causing gastrointestinal upset and predisposing patients to gastrointestinal bleeding, while ER-DP tends to be poorly tolerated because of headaches. In a post hoc analysis of the PRoFESS data comparing aspirin and ER-DP with clopidogrel, it was shown that there was a significantly higher withdrawal rate for patients on the combination therapy (18\% vs $12.5 \%$; $\mathrm{p}=0.006)^{16}$ (table 2). Clopidogrel can cause diarrhoea and rash, but is associated with less gastrointestinal haemorrhage than aspirin. Moreover, when aspirin and ER-DP are used in combination, there is a higher rate of haemorrhagic events as opposed to using clopidogrel alone (4.1\% vs 3.6\%, HR 1.15, 95\% CI 1.00

Table 1 Risk of recurrent ischaemic stroke and haemorrhagic complications in the ProFESS trial

\begin{tabular}{lllll}
\hline & $\begin{array}{l}\text { Events } \\
\text { on aspirin } \\
\text { and ER-DP }\end{array}$ & $\begin{array}{l}\text { Events on } \\
\text { clopidogrel }\end{array}$ & HR (95\% Cl) \\
\hline Risk of recurrent ischaemic stroke & $916(9.0 \%)$ & $898(8.8 \%)$ & $1.01(0.92$ to 1.11$)$ \\
Major haemorrhage & $419(4.1 \%)$ & $365(3.6 \%)$ & $1.15(1.00$ to 1.32$)$ \\
Intracranial haemorrhage & $147(1.4 \%)$ & $103(1.0 \%)$ & $1.42(1.11$ to 1.83$)$ \\
\hline
\end{tabular}

Adapted from Sacco et al.

ER-DP, extended-release dipyridamole; PRoFESS, Prevention Regimen for Effectively Avoiding Second Strokes.
Table 2 Recurrent stroke and withdrawal rates in patients with mild strokes enrolled in the ProFESS trial

\begin{tabular}{lccc}
\hline & $\begin{array}{l}\text { Events } \\
\text { on aspirin } \\
\text { and ER-DP }\end{array}$ & $\begin{array}{l}\text { Events on } \\
\text { clopidogrel }\end{array}$ & OR (95\% CI) \\
\hline $\begin{array}{l}\text { Recurrence of ischaemic stroke } \\
\text { (mild stroke subtype) at } 90 \text { days }\end{array}$ & $11(1.64 \%)$ & $20(2.91 \%)$ & $0.56(0.26$ to 1.18) \\
$\begin{array}{l}\text { Withdrawal from treatment } \\
\text { at } 90 \text { days }\end{array}$ & $121(18.0 \%)$ & $86(12.5 \%)$ & $1.51(1.12$ to 2.04) \\
\hline
\end{tabular}

Adapted from Bath et al. ${ }^{16}$

ER-DP, extended-release dipyridamole; PRoFESS, Prevention Regimen for Effectively Avoiding Second Strokes.

to $1.32 ; \mathrm{p}=0.057)$, and there are also more intracranial haemorrhages when compared with clopidogrel (1.4\% vs $1.0 \%$, HR 1.42 , $95 \%$ CI 1.11 to 1.83$)^{1}$ (table 1 ). Side effects more commonly reported with cilostazol as compared with aspirin were diarrhoea, palpitations, dizziness and tachycardia. ${ }^{2}$

\section{STROKE PREVENTION ANTIPLATELET THERAPY: WHAT IS THE VERDICT?}

\section{General atheromatous disease}

Patients with ischaemic stroke are likely to have concomitant occult and overt ischaemic heart disease, ${ }^{17}$ and are at risk of recurrent stroke and other cardiovascular events. ${ }^{18}$ Therefore, in order to prevent coronary as well as cerebrovascular events in such patients, it would make good clinical sense to choose a drug to protect the patient against recurrent stroke and events related to occult or overt ischaemic cardiac disease. Clopidogrel has an established place in the secondary prevention of coronary heart disease as shown in the Clopidogrel in Unstable Angina to Prevent Recurrent Events (CURE) trial, ${ }^{19}$ whereas similar evidence is lacking for ER-DP. The PRoFESS trial did show that there were similar cardiac event rates in both treatment groups (for myocardial infarction, HR $0.90,95 \%$ CI 0.73 to 1.10 ), but the follow-up only lasted a mean of 2.5 years, and as such patients are at risk of recurrent vascular events for many years, ${ }^{18}$ it will not be possible to determine if the combination therapy will be as effective as clopidogrel in the longer term. ${ }^{1}$ It is worth noting that patients with severe coronary artery disease and unstable angina were excluded from the PRoFESS trial, ${ }^{20}$ possibly due to lack of safety data for dipyridamole in patients with severe ischaemic heart disease.

Different stroke subtypes and transient ischaemic attacks (TIAs) The pathophysiology, natural history and mortality outcomes of lacunar and large artery ischaemic strokes are different. ${ }^{15}{ }^{21}$ As a result, the treatment and management of stroke should take account of its heterogeneous nature. While there is no direct evidence to show that clopidogrel is superior in large artery strokes, it has been shown that it is not inferior to aspirin and ER-DP combination therapy for all strokes. In light of this, clopidogrel should be the unanimous choice for the prevention of recurrent stroke, irrespective of stroke subtype. And because many stroke patients have concomitant ischaemic heart disease, they should be given clopidogrel, or even in some instances, aspirin and clopidogrel, if they have had a recent cardiac event as well. ${ }^{22}$ While there is no evidence base for the use of clopidogrel in TIAs, it may be just as effective as it is in secondary stroke prevention, as TIAs and stroke are part of the same disease spectrum. Cilostazol also has a role in secondary stroke prevention, and it would be interesting to compare it with other current antiplatelet agents. 


\section{Safety, efficacy, side effect profile and costs}

As cerebrovascular and coronary heart disease are closely related, it would be logical to choose a drug that is effective in preventing both recurrent coronary and cerebrovascular events. Clopidogrel seems to be the natural choice as it has shown efficacy in both cerebrovascular and coronary disease, and since it can be used safely in patients with co-existing severe coronary heart disease. Furthermore, clopidogrel is better tolerated, and therefore compliance is less likely to be an issue, as is observed in clinical practice. These costs may determine how widely it is used in the clinical context, but should not influence our prescribing practice. These costs can fluctuate greatly (such as when drugs become generic), and thus reasons for using certain drugs can change over time. As drug cost is very important in determining cost effectiveness, it is most helpful that a clinical and cost effectiveness analysis into these antiplatelet agents was recently published in a NICE technology appraisal. ${ }^{23}$ It found that generic clopidogrel was as effective and also more cost efficient when compared with ER-DP and aspirin in combination for patients with ischaemic stroke. It also recommended the use of clopidogrel for peripheral arterial and multivascular disease.

Although guidelines from the American Stroke Association on secondary stroke prevention suggested the use of aspirin or clopidogrel as monotherapy, or a combination of aspirin and ER-DP as the only acceptable options for initial therapy, ${ }^{24}$ with similar recommendations being given in European guidelines, ${ }^{25}$ it is worth considering the cost effectiveness argument recently presented by NICE, and using clopidogrel as the first antiplatelet of choice for those with a heavy burden of vascular disease.

We feel that both recurrent strokes and future coronary events can be prevented in patients with non-cardioembolic cerebrovascular ischaemic events and that a drug with proven efficacy and safety in both domains, such as clopidogrel, should be chosen. The AHA guidelines recommend that the choice of antiplatelet therapy should depend on the individual patient's characteristics. Therefore, clopidogrel would be the best choice in patients at risk of developing coronary heart disease.

\section{CONCLUSION}

Clopidogrel and combination antiplatelet therapy with aspirin and ER-DP are equally effective in preventing recurrent ischaemic stroke. Clopidogrel has the additional benefit that it can be used in patients with severe ischaemic heart disease, and is also better tolerated than the combination of aspirin and ERDP. Clopidogrel has a better side effect profile, and its generic preparation has been proven in the UK to be more cost effective

\section{Main messages}

- Stroke is a heterogeneous disease, with the different subtypes each having different pathophysiology, natural history and mortality.

- Cerebrovascular disease and ischaemic heart disease are closely related, with many patients having multivascular disease.

- Clopidogrel should be the first line antiplatelet for secondary prevention of ischaemic stroke as it has established efficacy in all stroke subtypes, in coronary heart disease and peripheral arterial disease.

\section{Current research questions}

As stroke is a heterogeneous disease, future research trials should be sufficiently powered to determine differences between various antiplatelet regimens in the different stroke subtypes.

- Further research into the antiplatelet agent cilostazol would help to determine its place in secondary stroke prevention.

- More trials investigating the secondary prevention of both stroke and ischaemic heart disease should be performed since all vascular disease is inter-related.

than aspirin and ER-DP in combination. Stroke patients have a huge burden of vascular disease, and are likely to be at risk of future cardiac and cerebral events. As clopidogrel has a strong evidence base showing it reduces recurrent cardiac events and prevents recurrent stroke, we should consider using it as first line therapy in patients with ischaemic stroke. We welcome the guidance produced by the American Stroke Association and the European Stroke Organisation on secondary stroke prevention using either aspirin or clopidogrel monotherapy or the combination of aspirin and ER-DP as initial management. However, the recently published NICE guidelines recommending the use of clopidogrel for preventing ischaemic stroke as well as multivascular disease are immensely helpful, and should encourage us to use this particular antiplatelet agent more aggressively for such patients. We feel that current guidelines for secondary stroke prevention should be reviewed in light of the information presented here, which reinforces clopidogrel as the first choice antiplatelet therapy for all ischaemic strokes and TIAs, to prevent further strokes and coronary events.

\section{Key references}

Sacco RL, Diener HC, Yusuf S, et al. Aspirin and extendedrelease dipyridamole versus clopidogrel for recurrent stroke. N Engl J Med 2008;359:1238-51.

- Bath PM, Cotton D, Martin RH, et al. Effect of combined aspirin and extended-release dipyridamole versus clopidogrel on functional outcome and recurrence in acute, mild ischemic stroke: PRoFESS subgroup analysis. Stroke 2010;41:732-8.

- Diener HC, Sacco R, Yusuf S. Rationale, design and baseline data of a randomized, double-blind, controlled trial comparing two antithrombotic regimens (a fixed-dose combination of extended-release dipyridamole plus ASA with clopidogrel) and telmisartan versus placebo in patients with strokes: the Prevention Regimen for Effectively Avoiding Second Strokes Trial (PRoFESS). Cerebrovasc Dis 2007;23:368-80.

- Furie KL, Kasner SE, Adams RJ, et al. Guidelines for the Prevention of Stroke in Patients with Stroke or Transient Ischemic Attack. A Guideline for Healthcare Professionals from the American Heart Association/American Stroke Association. Stroke 2011;42:227-76.

- European Stroke Organisation (ESO) Executive Committee, ESO Writing Committee. Guidelines for management of ischaemic stroke and transient ischaemic attack 2008. Cerebrovasc Dis 2008;25:457-507. 


\section{SELF-ASSESSMENT QUESTIONS (TRUE (T)/FALSE (F); ANSWERS AFTER THE REFERENCES)}

1. Patients with stroke are more likely to have occult cardiovascular disease.

2. Clopidogrel is superior to aspirin and extended-release dipyridamole for the prevention of recurrent ischaemic stroke.

3. Clopidogrel has proven efficacy when used in both cerebrovascular and coronary heart disease.

4. The risk of intracranial haemorrhage is higher with the use of clopidogrel as compared with aspirin and extended-release dipyridamole in combination.

5. Clopidogrel can be used as a treatment option in patients with peripheral arterial disease.

Competing interests None

Contributors DA wrote the manuscript with critical input and revisions from JCS.

Provenance and peer review Not commissioned; externally peer reviewed.

\section{REFERENCES}

1. Sacco RL, Diener HC, Yusuf S, et al. Aspirin and extended-release dipyridamole versus clopidogrel for recurrent stroke. N Engl J Med 2008;359:1238-51.

2. Shinohara Y, Katayama Y, Uchiyama S, et al. Cilostazol for prevention of secondary stroke (CSPS 2): an aspirin-controlled, double-blind, randomised non-inferiority trial. Lancet Neurol 2010;9:959-68.

3. Chen ZM, Sandercock P, Pan HC, et al. Indications for early aspirin use in acute ischemic stroke: A combined analysis of 40000 randomized patients from the Chinese Acute Stroke Trial and the International Stroke Trial. On behalf of the CAST and IST collaborative groups. Stroke 2000;31:1240-9.

4. Baigent C, Blackwell L, Collins R, et al. Aspirin in the primary and secondary prevention of vascular disease: collaborative meta-analysis of individual participant data from randomised trials. Lancet 2009;373:1849-60.

5. Diener HC, Cunha L, Forbes C, et al. European Stroke Prevention Study. 2. Dipyridamole and acetylsalicylic acid in the secondary prevention of stroke. J Neurol Sci 1996;143:1-13.

6. Halkes PH, van Gijn J, Kappelle LJ, et al. Aspirin plus dipyridamole versus aspirin alone after cerebral ischaemia of arterial origin (ESPRIT): randomised controlled trial. Lancet 2006;367:1665-73.

7. Hass WK, Easton JD, Adams HP Jr, et al. A randomized trial comparing ticlopidine hydrochloride with aspirin for the prevention of stroke in high-risk patients. Ticlopidine Aspirin Stroke Study Group. N Engl J Med 1989;321:501-7.

8. Gorelick PB, Richardson D, Kelly M, et al. Aspirin and ticlopidine for prevention of recurrent stroke in black patients: a randomized trial. JAMA 2003;289:2947-57.

9. CAPRIE Steering Committee. A randomised, blinded, trial of clopidogrel versus aspirin in patients at risk of ischaemic events (CAPRIE). Lancet 1996:348:1329-39.

10. Sudlow CL, Mason G, Maurice JB, et al. Thienopyridine derivatives versus aspirin for preventing stroke and other serious vascular events in high vascular risk patients. Cochrane Database Syst Rev 2009;(4):CD001246.

11. Diener HC, Bogousslavsky J, Brass LM, et al. Aspirin and clopidogrel compared with clopidogrel alone after recent ischaemic stroke or transient ischaemic attack in high-risk patients (MATCH): randomised, double-blind, placebo-controlled trial. Lancet 2004;364:331-7.

12. Bhatt DL, Fox KA, Hacke W, et al. Clopidogrel and aspirin versus aspirin alone for the prevention of atherothrombotic events. N Engl J Med 2006;354:1706-17.

13. Markus HS, Droste DW, Kaps M, et al. Dual antiplatelet therapy with clopidogrel and aspirin in symptomatic carotid stenosis evaluated using doppler embolic signal detection. The Clopidogrel and Aspirin for Reduction of Emboli in Symptomatic Carotid Stenosis (CARESS) Trial. Circulation 2005;111:2233-40.

14. Stroke Trials Registry - Triple Antiplatelets for Reducing Dependency after Ischaemic Stroke (TARDIS). 2010. http://www.strokecenter.org/trials/TrialDetail.aspx?tid=959 (accessed 8 Jul 2011).

15. Sharma JC, Vassallo M, Ross IN. ProFESS trial: any lessons to learn for future antiplatelet trials? Int J Stroke 2009;4:458-60.

16. Bath PM, Cotton D, Martin RH, et al. Effect of combined aspirin and extendedrelease dipyridamole versus clopidogrel on functional outcome and recurrence in acute, mild ischemic stroke: PRoFESS subgroup analysis. Stroke 2010;41:732-8.

17. Gongora-Rivera F, Labreuche J, Jaramillo A, et al. Autopsy prevalence of coronary atherosclerosis in patients with fatal stroke. Stroke 2007;38:1203-10.

18. Hankey GJ, Jamrozik K, Broadhurst RJ, et al. Five-year survival after first-ever stroke and related prognostic factors in the Perth Community Stroke Study. Stroke 2000;31:2080-6.

19. Yusuf S, Zhao F, Mehta SR, et al. Effects of clopidogrel in addition to aspirin in patients with acute coronary syndromes without ST-segment elevation. N Engl J Med 2001;345:494-502.

20. Diener HC, Sacco R, Yusuf S. Rationale, design and baseline data of a randomized double-blind, controlled trial comparing two antithrombotic regimens (a fixed-dose combination of extended-release dipyridamole plus ASA with clopidogrel) and telmisartan versus placebo in patients with strokes: the Prevention Regimen for Effectively Avoiding Second Strokes Trial (PRoFESS). Cerebrovasc Dis 2007;23:368-80

21. Jackson C, Sudlow C. Comparing risks of death and recurrent vascular events between lacunar and non-lacunar infarction. Brain 2005;128:2507-17.

22. Albers GW, Amarenco P. Combination therapy with clopidogrel and aspirin: can the CURE results be extrapolated to cerebrovascular patients? Stroke 2001:32:2948-9.

23. NICE TA210 Vascular disease-clopidogrel and dipyridamole: Guidance. 2010. http:// www.nice.org.uk/guidance/TA210 (accessed 3 Dec 2010).

24. Furie KL, Kasner SE, Adams RJ, et al. Guidelines for the prevention of stroke in patients with stroke or transient ischemic attack: a guideline for healthcare professionals from the American Heart Association/American Stroke Association. Stroke 2011; 42:227-76.

25. European Stroke Organisation (ESO) Executive Committee, ESO Writing Committee. Guidelines for management of ischaemic stroke and transient ischaemic attack 2008. Cerebrovasc Dis 2008;25:457-507. http://content.karger.com/ ProdukteDB/produkte. $a s p ?$ Aktion=ShowPDF\&ArtikeINr=131083\&Ausgabe $=$ 235865\&Produkt $\mathrm{Nr}=224153$ \&filename=131083.pdf (accessed 3 Dec 2010)

\section{ANSWERS}

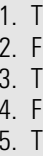

2. $\mathrm{F}$

3. $T$

4. $\mathrm{F}$

5. $\mathrm{T}$ 